\title{
Isolation, Structure, Absolute Stereochemistry and HIV-1 Integrase Inhibitory Activity of Integrasone, A Novel Fungal Polyketide
}

Kithsiri B. Herath, ${ }^{\dagger}$ Hiranthi Jayasuriya,${ }^{\dagger}$ Gerald F. Bills,,${ }^{\ddagger}$ Jon D. Polishook, ${ }^{\dagger}$ Anne W. Dombrowski, ${ }^{\dagger}$ Ziqiang Guan, ${ }^{\dagger}$ Peter J. Felock, ${ }^{\mathbb{I}}$ Daria J. Hazudaף and Sheo B. Singh ${ }^{\dagger *}$

†Natural Products Chemistry, Merck Research Laboratories, P. O. Box 2000, Rahway, New Jersey 07065 (USA), ¥CIBE, Merck Sharp \& Dohme de Espana, S. A. Josefa Valcárcel 38, 28027 Madrid, Spain and "Department of Antiviral Research, Merck Research Laboratories, West Point, Pennsylvania 19486 (USA)

Supporting Information Available: Copies of ${ }^{1} \mathrm{H},{ }^{13} \mathrm{C}$ NMR, COSY, TOCSY and NOESY spectral data (5 pages). This material is available free of charge via the Internet at http://pubs.acs.org.

* To whom correspondence should be addressed. Fax: (732) 594-6880. E-mail: sheo_singh@merck.com 


\section{$500 \mathrm{MHz}{ }^{1} \mathrm{H}$ NMR spectrum of integrasone in $\mathrm{CD}_{3} \mathrm{OD}$}

zzssiaztuzazz.

10 ng in mathanol

K. Berat:

Fab14/0

Integraf sctive

soonhr

Kithair Hezath, Pab 142000

HI 1D-Nifir Spectrun : 0 nin

solvent CD3OD Tenp: 25,0

Varian 00.0 :

Mot 79

Nal-DG: nane

DEX: 'npre'

$\operatorname{aum} / \mathrm{Ix}_{\mathrm{s}} \mathrm{es}$ 'nene'

ap 0.57 WD 4.701 Don

vs 52

$\mid$
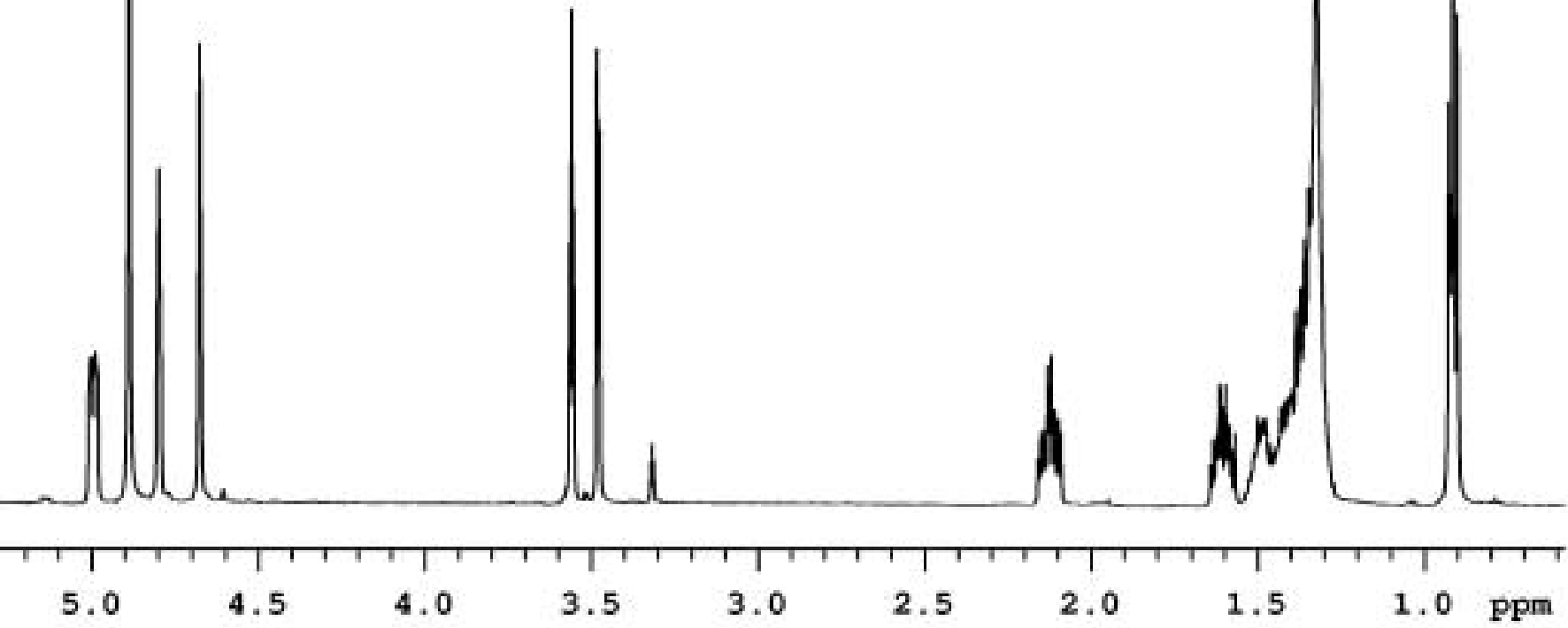


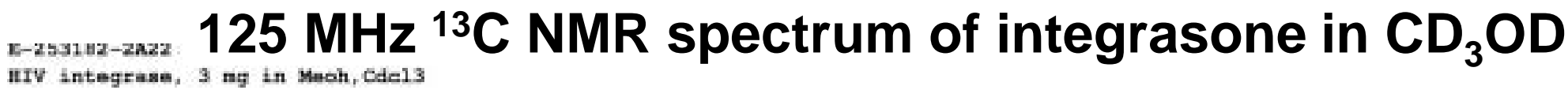

Kuthsiri_Herath, Fob 102000

c13 1D-kMR spectrun its nin

Solvent: CD3OD

Varien 500,0 :

900473

$\mathrm{NB}-\mathrm{DC}$ : ' nose'

PEX: 'none'

and /fraci 'none'

ag 0,896 wp $167,662 \mathrm{ppn}$

val 47

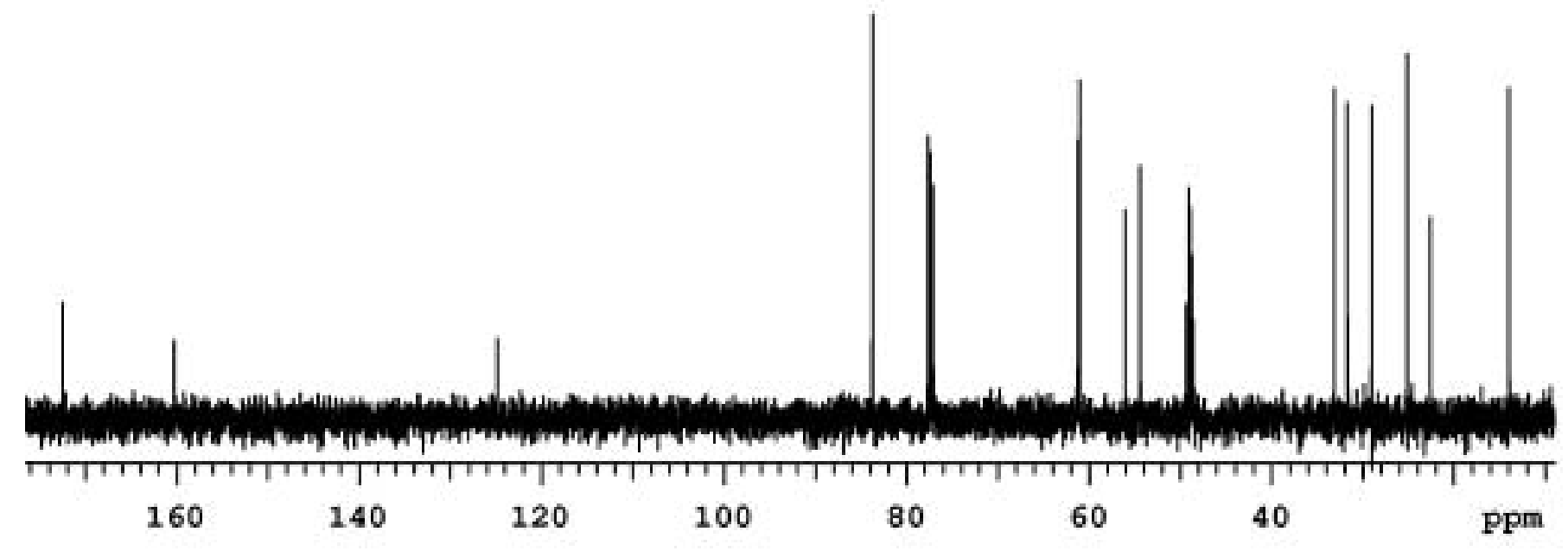




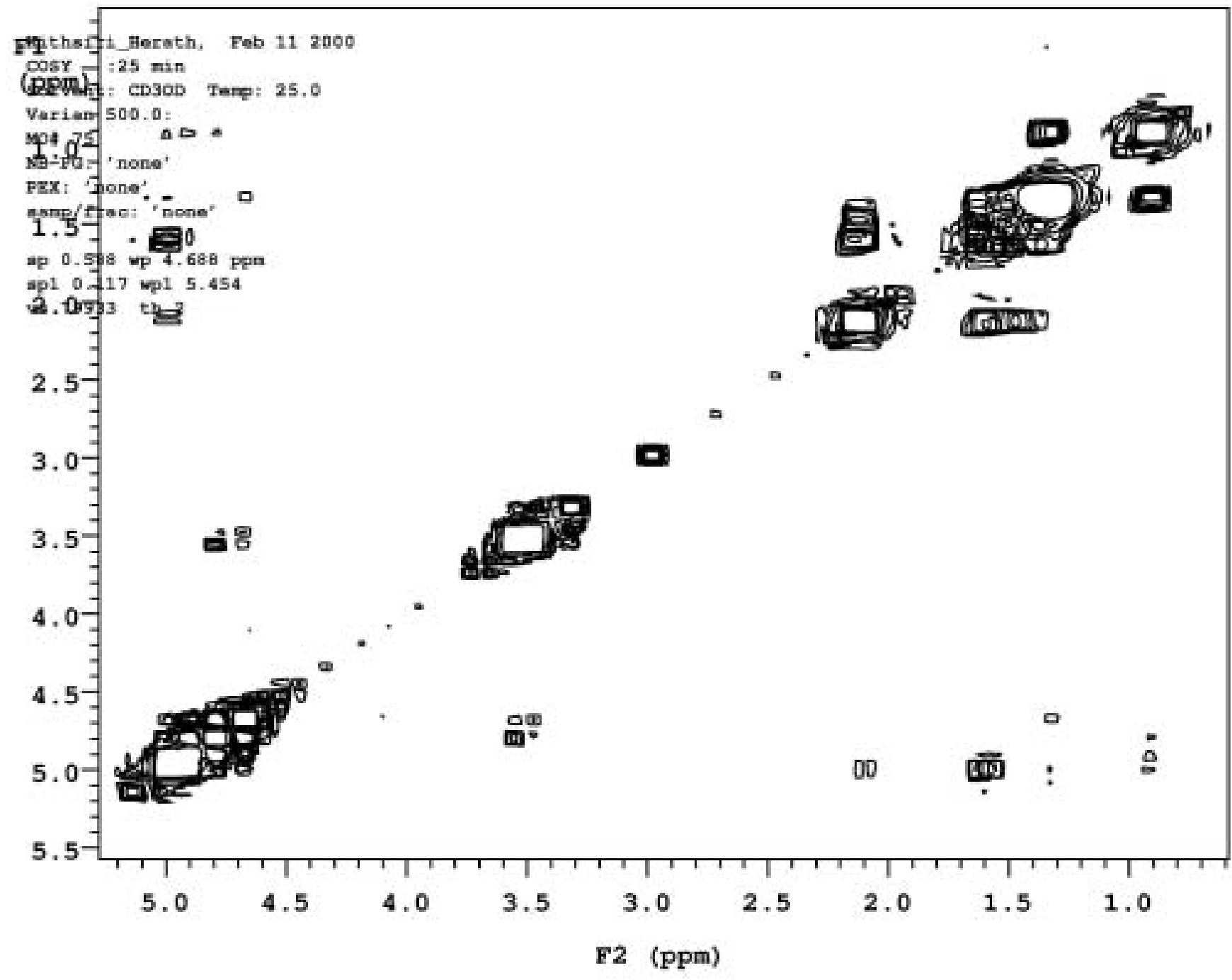


E-2331日1-2az2 $10 \mathrm{mg}$ in $\mathrm{MeOH}$

$500 \mathrm{MHz}$ TOCSY spectrum of integrasone in $\mathrm{CD}_{3} \mathrm{OD}$ integraan

500 mha

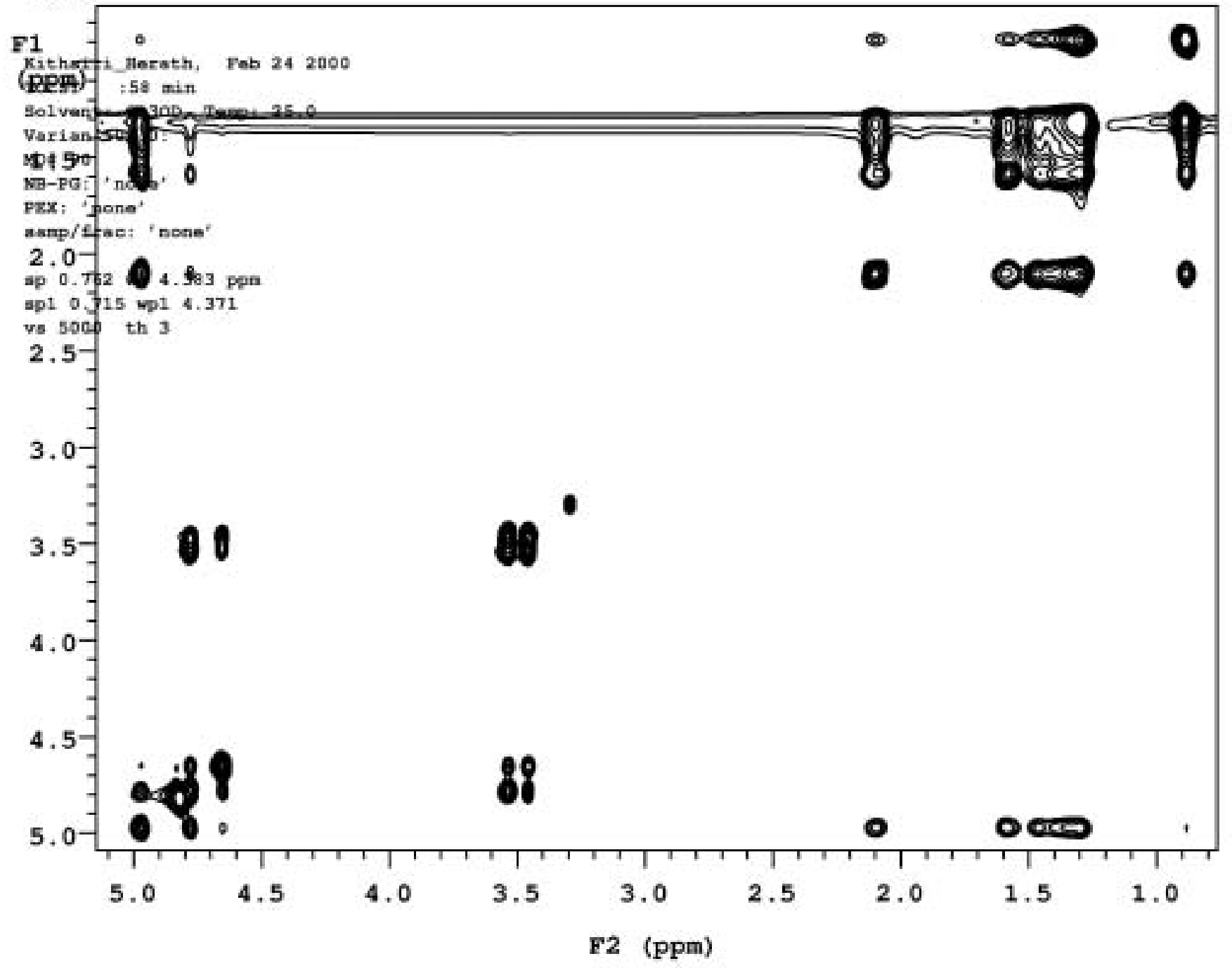




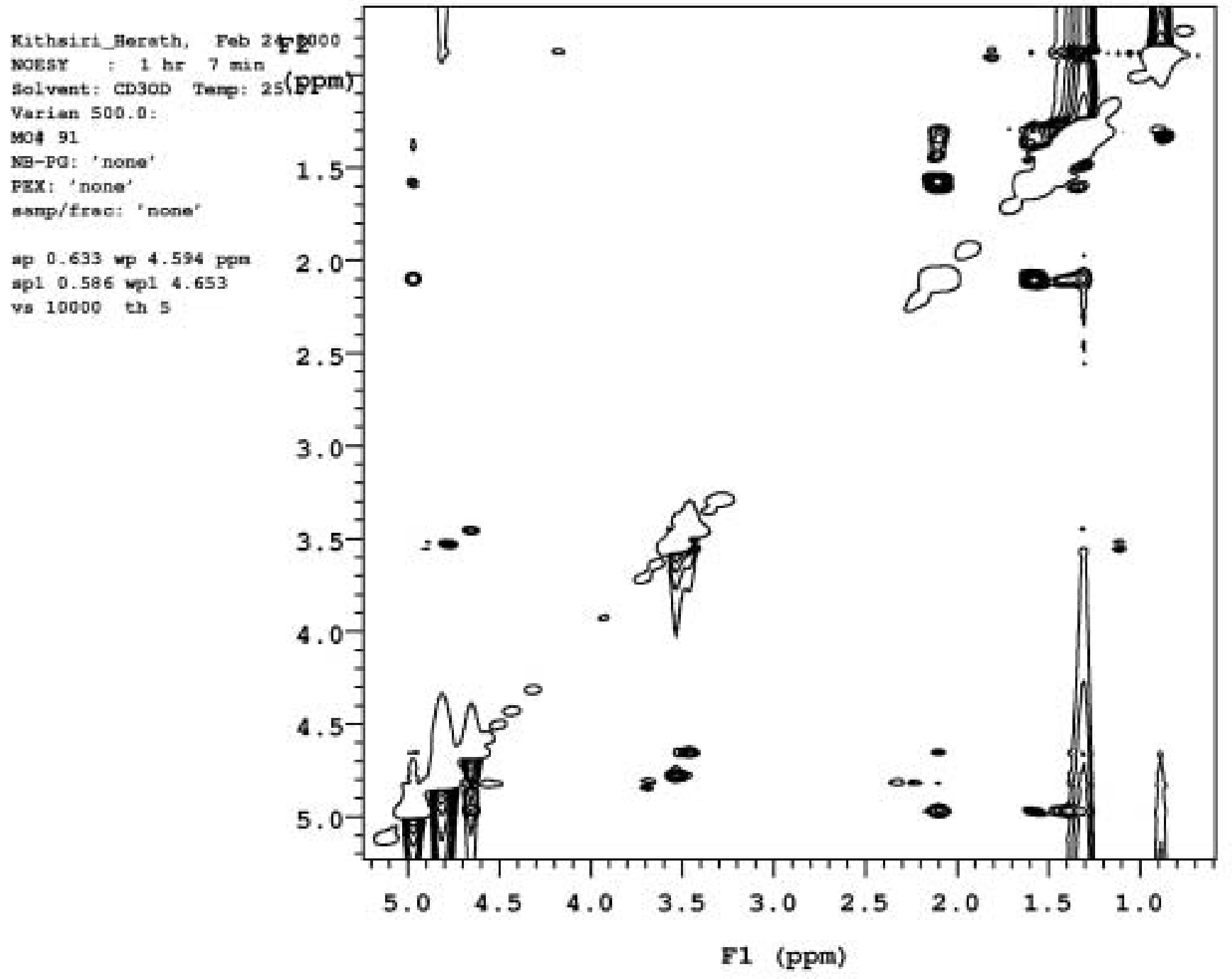

